Conclusion Although there are many surgical options available for management of CVF, only a few case reports exist regarding non-surgical management of CVF. We recommend that combined endoscopic management of CVF should be considered in patients who are too frail for surgery or have multiple co-morbidities.

\section{PTH-072 ENDOSCOPIC MANAGEMENT OF PHYTOBEZOAR IN PATIENT WITH PRADER-WILLI SYNDROME}

Faisal Nawaz* , Nasima Ali, Monzur Ahmed. Good Hope Hospital, Birmingham, UK

\subsection{6/gutjnl-2019-BSGAbstracts.97}

Introduction Prader-Willi syndrome (PWS) was first described in 1956 and is now recognised as a multisystemic complex genetic disorder caused by lack of expression of genes on the paternally inherited chromosome 15 . Clinical features include hyperphagia, obesity and diabetes. A phytobezoar is a type of bezoar, or trapped mass in the gastrointestinal tract, that consists of indigestible plant material. We report a case of phytobezoar due to hyperphagia in a patient with PWS, which was successfully treated endoscopically.

Methods A 22 year old man with PWS was admitted with a one day history of severe abdominal pain associated with distention and nausea but no vomiting. He was opening his bowels and passing wind. He had a history of anxiety and had spinal surgery for scoliosis at age 10 years. He was a vegetarian and had been living in a residential home for three years where his meals were regulated. On examination, he was not obese (BMI $20 \mathrm{~kg} / \mathrm{m}^{\wedge}$ 2) and had a distended, tender abdomen. Routine blood tests $(\mathrm{FBC}, \mathrm{U}+\mathrm{E})$ were normal. Initial abdominal x-ray showed a very distended stomach containing food material. An abdominal CT scan confirmed these findings and worryingly the report mentioned 'imminent danger of perforation.' The surgeons reviewed the patient and referred him for possible endotherapy prior to considering surgery. Hence, a gastroscopy was performed under conscious sedation using a twin channel gastroscope (Olympus GIF 2T200). A large phytobezoar was seen in the stomach. There was no gastric necrosis or pyloric obstruction and the duodenum was normal. The phytobezoar was softened with water and fragmented into smaller pieces with a $3 \mathrm{~cm}$ Trapezoid basket (Boston Scientific). The smaller pieces of the phytobezoar were sucked out slowly using the large channel gastroscope. Eventually the entire phytobezoar was removed in this manner; the whole procedure taking 90 minutes.

Results The patient tolerated the procedure well and was discharged after a day of observation.

Conclusion Gastric bezoars can cause outlet obstruction, ulceration, and subsequent bleeding, whereas small intestinal bezoars present with small bowel obstruction and ileus. The currently available treatment options for a gastric phytobezoar include dissolution of the bezoar by Coca-Cola ${ }^{\circledR}$, removal by endoscopic devices, laparotomy, and laparoscopic surgery. Gastric rupture and necrosis are a known cause of mortality in patients with PWS. Hence, timely endoscopic management can help avoid these complications and the need for surgery.

\section{PTH-073 SUCCESSFUL ENDOSCOPIC CLOSURE OF DU PERFORATION USING OTSC IN A FRAIL PATIENT UNSUITABLE FOR SURGERY}

Mathilda Sutherland*, Kohilan Gananandan, Cameron Green, Sudeep Tanwar. Whipps Cross University Hospital, Barts Health NHS Trust, London, UK

\subsection{6/gutjnl-2019-BSGAbstracts.98}

Introduction Endoscopic closure devices continue to be used successfully to close iatrogenic perforations within the GI tract. However, only a handful of cases describe successful closure of a spontaneous duodenal perforation. We present the first UK case to demonstrate successful endoscopic closure of perforated duodenal ulcer using over-the-scope clips in a patient unfit for surgery.

Methods The case involved an 85-year-old bedbound female patient with COPD, mild dementia, hypertension, hypercholesterolaemia and three previous cardiac arrests. She attended hospital unwell after passing melaena and experiencing abdominal pain. Her abdomen was markedly tender and subsequent CT imaging confirmed a full thickness perforation at the duodenal bulb. Due to her multiple medical comorbidities she was not an operative candidate and the perforation would be managed conservatively with bowel rest and broad spectrum antibiotics. The patient was referred to the gastroenterology team who considered high risk endoscopic closure after a discussion with the patient's family. After transfusion and fluid resuscitation, gastroscopy with $\mathrm{CO}_{2}$ insufflation was performed under conscious sedation (1 mg midazolam and $50 \mathrm{mcg}$ fentanyl). A deep ulcer with evidence of recent bleeding was identified in the duodenal bulb, through which the full thickness perforation site could be observed. Two OTSC clips were applied successfully to close the defect.

Results Following OTSC clip deployment the patient was kept nil by mouth. Seventy-two hours post endotherapy an interval CT identified a reduced volume of pneumoperitoneum with no extraluminal leakage of contrast confirming successful closure. The patient was then allowed to eat and drink normally. A repeat gastroscopy on day seven demonstrated full closure of the duodenal perforation. The patient was commenced on H. pylori eradication and discharged eleven days after initial presentation.

Conclusions Surgical repair remains the gold standard treatment for the repair of a spontaneous enteral perforations. Candidacy for surgical repair does however require candidacy for general anaesthesia which is often not the case amongst our increasingly aging and comorbid population. This is one of a handful of cases worldwide and indeed the first ever reported case in the UK of a spontaneous duodenal perforation being successfully repaired using an endoscopic closure device. The key determinant for success in this case were the use of $\mathrm{CO}_{2}$ insufflation and the prompt timing of endoscopic repair which obviated gross peritoneal contamination. Whilst this proof of concept case confirms that endoscopic repair is possible in this context, further studies are required to determine if this modality has any role in patients in whom surgical repair is not contraindicated. 